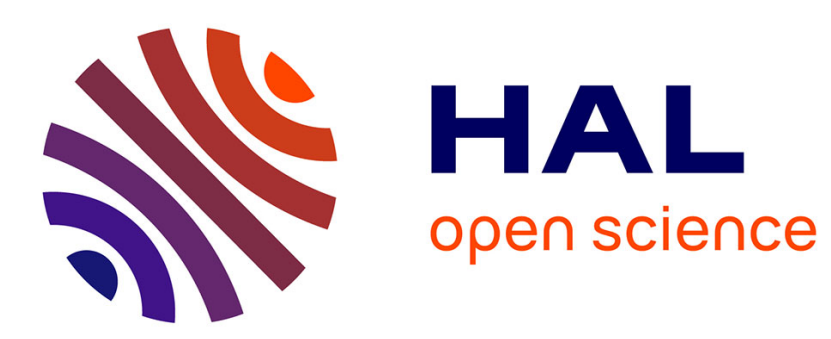

\title{
Improved Cryptanalysis of the KMOV Elliptic Curve Cryptosystem
}

Abderrahmane Nitaj, Willy Susilo, Joseph Tonien

\section{To cite this version:}

Abderrahmane Nitaj, Willy Susilo, Joseph Tonien. Improved Cryptanalysis of the KMOV Elliptic Curve Cryptosystem. Provable Security ProvSec 2019, 2019, Cairns, Australia. 10.1007/978-3-03031919-9_12 . hal-02320906

\section{HAL Id: hal-02320906 \\ https://hal-normandie-univ.archives-ouvertes.fr/hal-02320906}

Submitted on 19 Oct 2019

HAL is a multi-disciplinary open access archive for the deposit and dissemination of scientific research documents, whether they are published or not. The documents may come from teaching and research institutions in France or abroad, or from public or private research centers.
L'archive ouverte pluridisciplinaire HAL, est destinée au dépôt et à la diffusion de documents scientifiques de niveau recherche, publiés ou non, émanant des établissements d'enseignement et de recherche français ou étrangers, des laboratoires publics ou privés. 


\title{
Improved Cryptanalysis of the KMOV Elliptic Curve Cryptosystem
}

\author{
Abderrahmane Nitaj ${ }^{1}$, Willy Susilo ${ }^{2}$, and Joseph Tonien ${ }^{2}$ \\ 1 LMNO, Université de Caen Normandie, France \\ abderrahmane.nitaj@unicaen.fr \\ 2 Institute of Cybersecurity and Cryptology, School of Computing and Information \\ Technology, University of Wollongong, Australia \\ \{willy.susilo, joseph.tonien\}@uow.edu.au
}

\begin{abstract}
This paper presents two new improved attacks on the KMOV cryptosystem. KMOV is an encryption algorithm based on elliptic curves over the ring $\mathbb{Z}_{N}$ where $N=p q$ is a product of two large primes of equal bit size. The first attack uses the properties of the convergents of the continued fraction expansion of a specific value derived from the KMOV public key. The second attack is based on Coppersmith's method for finding small solutions of a multivariate polynomial modular equation. Both attacks improve the existing attacks on the KMOV cryptosystem.
\end{abstract}

\section{Introduction}

The RSA cryptosystem [21, invented in 1978 by Rivest, Shamir and Adleman, is the most widely used cryptosystem. The main parameters in RSA are two integers, the RSA modulus $N=p q$ where $p$ and $q$ are large prime numbers, and the public exponent $e$, which is an integer satisfying $\operatorname{gcd}(e,(p-1)(q-1))=1$. The private exponent is the integer $d$ satisfying $e d \equiv 1(\bmod (p-1)(q-1))$. In many implementations, the private exponent $d$ is required to be small to ease decryption and signature. Unfortunately, this scenario is dangerous and can be used to break the system [36]. In 1990, Wiener [25/24] presented an attack to break the RSA system if the private exponent $d$ satisfies $d<\frac{1}{\sqrt[4]{18}} N^{\frac{1}{4}}$. Since then, Wiener's bound has been extended in many situations, mainly by Boneh and Durfee [2] to $d<N^{0.292}$.

In 1985, Miller [17] and Koblitz [13] independently proposed to use elliptic curves in cryptography. Since then, many cryptosystems have been proposed based on elliptic curves. In the direction of RSA, Koyama, Maurer, Okamoto and Vanstone 14 proposed a cryptosystem, called KMOV, based on the elliptic curve $E_{N}(0, b)$ where $N=p q$ is an RSA modulus and $E_{N}(0, b)$ is the set of solutions of the modular equation $y^{2} \equiv x^{3}+b(\bmod N)$, together with the point at infinity, denoted $\mathcal{O}$. When the prime factors $p$ and $q$ are such that $p \equiv q \equiv 2$ $(\bmod 3)$, then any point $P \in E_{N}(0, b)$ satisfies $(p+1)(q+1) P=\mathcal{O}$. In KMOV, the public key is a pair $(N, e)$ where $N=p q$ with two prime integers satisfying $p \equiv q \equiv 2(\bmod 3)$ and $e$ is an integer satisfying $\operatorname{gcd}(e,(p+1)(q+1))=1$. The decryption exponent is the integer $d$ such that $e d \equiv 1(\bmod (p+1)(q+1))$. 
Notice that the modular equation $e d \equiv 1(\bmod (p+1)(q+1))$ is equivalent to the integer key equation $e d-k(p+1)(q+1)=1$. In 1995, Pinch [20] used the key equation and extended Wiener's attack to KMOV. He showed that one can factor the modulus $N=p q$ if $d<\frac{1}{3} N^{\frac{1}{4}}$. In [11, Ibrahimpasic extended the attack of Pinch by a few bits using an exhaustive search. Both attacks use the convergents of the continued fraction expansion of $\frac{e}{N}$. In [18, Nitaj considered the generalized equation $e u-(p+1)(q+1) v=w$ and showed that one can factor the modulus $N=p q$ if the parameters $u, v, w$ satisfy some specific conditions, especially if $u v<\frac{\sqrt{2} \sqrt{N}}{12}$. The method combines the continued fraction algorithm [4]7] and Coppersmith's method 8 for solving univariate modular equations.

In this paper, we extend the former attacks on KMOV. In the first attack we consider the KMOV key equation $e d-k(p+1)(q+1)=1$ and instead of using the convergents of $\frac{e}{N}$, we use the convergents of $\frac{e}{N+1+\left(1+\frac{3 \sqrt{2}}{4}\right) N^{\frac{1}{2}}}$. As a consequence, we show that one can factor the modulus $N=p q$ if the private exponent $d$ is such that $d<2 \sqrt{2} \frac{N^{\frac{3}{4}}}{\sqrt{e}}$. This bound improves the former bound $d<\frac{1}{3} N^{\frac{1}{4}}$, especially when the public exponent $e$ is significantly smaller then $N$.

In the second attack we consider the generalized key equation $e u-(p+1)(q+$ 1) $v=w$ and transform it to the modular polynomial equation $v(p+q+1)+N v+$ $w \equiv 0(\bmod e)$. We consider the polynomial $f(x, y, z)=x y+N x+z$ and apply Coppersmith's method to find the small solutions of the modular polynomial equation $f(x, y, z) \equiv 0(\bmod e)$. When $e=N^{\beta}, u<N^{\delta}$ and $|w|<N^{\gamma}$, if

$$
\delta<\frac{7}{6}-\gamma-\frac{1}{3} \sqrt{6 \beta-6 \gamma+1}-\varepsilon,
$$

where $\varepsilon$ is a small constant, then Coppersmith's method enables us to find $p+$ $q+1$, which combined with $N=p q$ gives $p$ and $q$. We note that in the standard situation of a KMOV instance with $e \approx N$ and $e u-(p+1)(q+1) v=1$, our new bound is $\delta<0.284$ which is much larger than the existing bounds.

The rest of this paper is organized as follows. In Section 2, we give some preliminaries on Coppersmith's method, continued fractions, elliptic curves and recall the KMOV cryptosystem. In Section 3, we present our first attack on KMOV based on continued fractions. In Section 4, we present our second attack on KMOV which is based on Coppersmith's method. We conclude the paper in Section 5.

\section{Preliminaries}

In this section, we give some preliminaries on Coppersmith's methods for solving modular polynomial equations, continued fractions and elliptic elliptic curves. For completeness, we recall the KMOV cryptosystem. 


\subsection{Coppersmith's method}

One of the difficult problems in algebra is to solve modular polynomial equations of the form

$$
f\left(x_{1}, \ldots, x_{n}\right) \equiv 0 \quad(\bmod e),
$$

where $f\left(x_{1}, \ldots, x_{n}\right) \in \mathbb{Z}\left[x_{1}, \ldots, x_{n}\right]$ is multivariate polynomial. In 1996, Coppersmith 8 introduced a rigorous method for finding the small solutions of the univariate polynomial equation $f(x) \equiv 0(\bmod e)$ and the small roots of the bivariate polynomial equation $f(x, y)=0$. Coppersmith's method is based on lattice reduction and is useful in cryptography, especially for attacking the RSA cryptosystem (see [16/5|1/19]). Since then, numerous variants of Coppersmith's method have been presented for multivariate polynomial equations assuming certain hypothesis. The following result of Howgrave-Graham [10] is useful for solving the polynomial equations.

Theorem 1 (Howgrave-Graham). Let e be a positive integer and $h(x, y, z) \in$ $\mathbb{Z}[x, y, z]$ be a polynomial with at most $\omega$ monomials. Let $m$ be a positive integer. Suppose that

$$
\begin{aligned}
& h\left(x_{0}, y_{0}, z_{0}\right) \equiv 0 \quad\left(\bmod e^{m}\right) \quad \text { and } \\
& \|h(x X, y Y, z Z)\|=\sqrt{\sum_{i, j, k} a_{i, j, k} x^{i} y^{j} z^{k}}<\frac{e^{m}}{\sqrt{\omega}},
\end{aligned}
$$

where $\left|x_{0}\right|<X,\left|y_{0}\right|<Y,\left|z_{0}\right|<Z$. Then $h\left(x_{0}, y_{0}, z_{0}\right)=0$ holds over the integers.

For a multivariate polynomial modular equation $f(x, y, z) \equiv 0(\bmod e)$, the idea in Coppersmith's method is to build certain modular polynomials equations $h(x, y, z) \equiv 0\left(\bmod e^{m}\right)$ sharing the modular solution $\left(x_{0}, y_{0}, z_{0}\right)$. These polynomials are generally built by applying Jochemz-May [12] method and applying lattice reduction techniques such as the LLL algorithm [15]. The LLL algorithm acts on lattices and the following result is useful (see [15]16[12]).

Theorem 2 (LLL). Let $\mathcal{L}$ be a lattice spanned by a basis $\left(u_{1}, \ldots, u_{\omega}\right)$, then the $L L L$ algorithm produces a new basis $\left(b_{1}, \ldots, b_{\omega}\right)$ satisfying

$$
\left\|b_{1}\right\| \leq \ldots \leq\left\|b_{i}\right\| \leq 2^{\frac{\omega(\omega-1)}{4(\omega+1-i)}} \operatorname{det}(\mathcal{L})^{\frac{1}{\omega+1-i}}, \quad i=1, \ldots, \omega .
$$

To find the root $\left(x_{0}, y_{0}, z_{0}\right)$, we use a system with three polynomial equations $h_{i}(x, y, z)=0, i=1,2,3$. By using Gröbner basis computation or resultant techniques, the system can be solved under the following widely believed assumption.

Assumption 1 The polynomials $h_{1}, h_{2}, h_{3} \in \mathbb{Z}[x, y, z]$ that are derived from the reduced basis of the lattice in Coppersmith's method are algebraically independent. 


\subsection{Continued fractions}

Let $\xi \neq 0$ be real number. The continued fraction expansion of $\xi$ is an expression of the form

$$
\xi=a_{0}+\frac{1}{a_{1}+\frac{1}{a_{2}+\frac{1}{\ddots}}},
$$

where $a_{0}$ is an integer and for $i \geq 1, a_{i}$ is a positive integer. The integers $a_{i}$, $i \geq 0$ are the partial quotients of the continued fraction expansion. The process to compute the integers $a_{i}$ for $i \geq 0$ is the continued fraction algorithm. The starting term is $x_{0}=\xi$ and for $i \geq 0$,

$$
a_{i}=\left\lfloor x_{i}\right\rfloor, x_{i+1}=\frac{1}{x_{i}-a_{i}} .
$$

When the continued fraction expansion is used with the first $k+1$ partial quotients, the fraction is a convergent. The following method is very useful for computing the convergents of $\xi$.

Theorem 3. The $k^{\text {th }}$ convergent can be determined as $\left[a_{0}, \ldots, a_{k}\right]=\frac{p_{k}}{q_{k}}$, where the sequences $\left\{p_{n}\right\}$ and $\left\{q_{n}\right\}$ are specified as follow $\}^{3}$;

$$
\begin{array}{rlll}
p_{-2}=0, & p_{-1}=1, & p_{n}=a_{n} p_{n-1}+p_{n-2}, & \forall n \geq 0, \\
q_{-2}=1, & q_{-1}=0, & q_{n}=a_{n} q_{n-1}+q_{n-2}, & \forall n \geq 0 .
\end{array}
$$

There are many properties related to the theory of continued fractions. One of the most important results is Legendre's Theorem (see Theorem 184 of [9]).

Theorem 4. Let $\xi \neq 0$ be a real number and $a, b$ be two positive integers such that $\frac{a}{b} \notin \mathbb{N}$ and $(a, b)=1$. If

$$
0<\left|\xi-\frac{a}{b}\right|<\frac{1}{2 b^{2}}
$$

then $\frac{a}{b}$ is a convergent of the continued fraction of $\xi$.

Note that computing a convergent $\frac{a}{b}$ of $\xi$ with the continued fraction algorithm is done in polynomial time in $\log (b)$.

\section{$2.3 \quad$ Elliptic curves}

Let $p \geq 5$ be a prime number and $a$ and $b$ two integers satisfying $4 a^{3}+27 b^{2} \not \equiv 0$ $(\bmod p)$. An elliptic curve $E_{p}(a, b)$ over $\mathbb{F}_{p}=\mathbb{Z} / p \mathbb{Z}$ is the set of solutions $(x, y) \in$ $\mathbb{F}_{p}^{2}$ satisfying the equation

$$
E_{p}(a, b): \quad y^{2} \equiv x^{3}+a x+b \quad(\bmod p),
$$

\footnotetext{
3 The convergents start with $\frac{p_{0}}{q_{0}}$, but it is a convention to extend the sequence index to -1 and -2 to allow the recursive formula to hold for $n=0$ and $n=1$
} 
together with a point $\mathcal{O}$, called the point at infinity. If $P_{1}=\left(x_{1}, y_{1}\right)$ and $P_{2}=$ $\left(x_{2}, y_{2}\right)$ are two points, then one have the following properties.

$-P_{1}+\mathcal{O}=\mathcal{O}+P_{1}=P_{1}$.

- The opposite of $P_{1}$ is $-P_{1}=\left(x_{1},-y_{1}\right)$.

- If $P_{2}=-P_{1}$, then $P_{1}+P_{2}=\mathcal{O}$.

- If $P_{2} \neq-P_{1}$, then $P_{1}+P_{2}=P_{3}=\left(x_{3}, y_{3}\right)$ where

$$
x_{3} \equiv \lambda^{2}-x_{1}-x_{2} \quad(\bmod p), \quad y_{3} \equiv \lambda\left(x_{1}-x_{3}\right)-y_{1} \quad(\bmod p),
$$

with

$$
\lambda= \begin{cases}\frac{y_{2}-y_{1}}{x_{2}-x_{1}} & \text { if } x_{1} \neq x_{2}, \\ \frac{3 x_{1}^{2}+a}{2 y_{1}} & \text { if } x_{1}=x_{2} .\end{cases}
$$

With the former addition law, the set $E_{p}(a, b)$ is a group of finite order $\# E_{p}(a, b)$ where $\# E_{p}(a, b)$ is the number of solutions $(x, y) \in \mathbb{F}_{p}^{2}$ of the equation (1) together with the point at infinity. According to a famous Theorem of Hasse (see [23], Chapter 5), we have $\# E_{p}(a, b)=p+1-t_{p}$, with $\left|t_{p}\right|<2 \sqrt{p}$, which is close to $p+1$, up to a small value $t_{p}$.

For specific values of $p, \# E_{p}(a, b)$ can be explicitly computed as for $p \equiv 2$ $(\bmod 3)($ see $[22])$.

Theorem 5. Let $E_{p}(0, b)$ be an elliptic curve over $\mathbb{F}_{p}$ with equation $y^{2} \equiv x^{3}+b$ $(\bmod p)$. If $p \equiv 2(\bmod 3)$, then number of points on $E_{p}(0, b)$ is $\# E_{p}(0, b)=$ $p+1$.

Since $\# E_{p}(a, b)$ is the order of the group $E_{p}(0, b)$ for the addition law, then $\# E_{p}(a, b) \cdot P=\mathcal{O}$ for any point $P \in E_{p}(a, b)$. When $p \equiv 2(\bmod 3)$, then for any point $P \in$, we have $(p+1) P=\mathcal{O}$. When $N$ is a composite square free integer and $a$ and $b$ are integers satisfying $4 a^{3}+27 b^{2} \not \equiv 0(\bmod p)$, one can define an elliptic curve $E_{N}(a, b)$ over the ring $\mathbb{Z} / N \mathbb{Z}$ by the equation

$$
E_{N}(a, b): \quad y^{2} \equiv x^{3}+a x+b \quad(\bmod N),
$$

together with a point $O$ at infinity. An addition law can be defined over $E_{N}(a, b)$ by using the same rules as the addition law on $E_{p}(a, b)$ by replacing modulo $p$ by modulo $N$. When the division by $x_{2}-x_{1}$ is not possible, this means that $\operatorname{gcd}\left(x_{2}-\right.$ $\left.x_{1}, n\right) \neq 1$. Since $0<\left|x_{2}-x_{1}\right|<n$, then $\operatorname{gcd}\left(x_{2}-x_{1}, n\right)=p \operatorname{or} \operatorname{gcd}\left(x_{2}-x_{1}, n\right)=q$. If $N=p q$ is an RSA modulus, this is equivalent to factoring $N$. Since the integer factorization problem is very hard, especially for RSA moduli, then the scenario that the addition does not exist is unlikely to happen. By the Chinese remainder theorem, every point $P=(x, y) \in E_{N}(a, b)$ is uniquely represented by a pair of points $\left(P_{p}, P_{q}\right) \in E_{p}(a, b) \times E_{q}(a, b)$ with the convention that $O$ is represented by the pair of points at infinity $\left(\mathcal{O}_{p}, \mathcal{O}_{q}\right) \in E_{p}(a, b) \times E_{q}(a, b)$. It follows that for $p \equiv q \equiv 2(\bmod 3)$ and for any point $P \in E_{N}(0, b)$, we have

$$
(p+1)(q+1) P=(p+1)(q+1)\left(P_{p}, P_{q}\right)=\left(\mathcal{O}_{p}, \mathcal{O}_{q}\right)=\mathcal{O} .
$$




\subsection{The KMOV Cryptosystem}

In 1991, Koyama, Maurer, Okamoto and Vanstone proposed a cryptosystem, called KMOV, based on the elliptic curve $E_{N}(0, b)$ where $N=p q$ is an RSA modulus. The scheme works as follows.

\section{- KMOV Key Generation algorithm.}

1. Choose two distinct prime numbers $p$ and $q$ of similar bit-length with $p \equiv q \equiv 2(\bmod 3)$.

2. Compute $N=p q$.

3. Choose $e$ such that $\operatorname{gcd}(e,(p+1)(q+1))=1$.

4. Compute $d=e^{-1}(\bmod (p+1)(q+1))$.

5. Keep $p, q, d$ secret, publish $N, e$.

- KMOV Encryption algorithm.

1. For a message $m=\left(m_{x}, m_{y}\right) \in \mathbb{Z}_{N}^{2}$, compute $b=m_{y}^{2}-m_{x}^{3}(\bmod N)$.

2. Compute the point $\left(c_{x}, c_{y}\right)=e\left(m_{x}, m_{y}\right)$ on the elliptic curve with equation $y^{2} \equiv x^{3}+b(\bmod N)$. The ciphertext is $c=\left(c_{x}, c_{y}\right)$.

\section{- KMOV Decryption algorithm.}

1. For a ciphertext $c=\left(c_{x}, c_{y}\right) \in \mathbb{Z}_{N}^{2}$, compute $b=c_{y}^{2}-c_{x}^{3}(\bmod N)$.

2. Compute the point $\left(m_{x}, m_{y}\right)=d\left(c_{x}, c_{y}\right)$ on the elliptic curve $y^{2} \equiv x^{3}+b$ $(\bmod N)$. The plaintext is $m=\left(m_{x}, m_{y}\right)$.

The complexity of the encryption and decryption algorithms are based on the size of the encryption key $e$ and the size of decryption key $d$, respectively. In a cryptosystem with a limited resource such as a credit card, it is desirable to have a smaller value of $d$ or $e$. Unfortunately, when $d$ is too small, Pinch [20] showed that one can factor the RSA modulus $N=p q$ if $d<\frac{1}{3} N^{\frac{1}{4}}$. Using a generalized attack, Nitaj [18] showed that one can factor $N$ when $d \equiv \frac{y}{x}(\bmod (p+1)(q+1))$ is much larger under some extra conditions on $x$ and $y$.

\section{A New Improved Attack Based on Continued Fractions}

In this section, we give an improved attack on KMOV based totally on the continued fraction algorithm.

\subsection{The new attack based on continued fractions}

The attacks presented in [20] and [11 take advantage on using the convergents of the continued fraction expansion of $\frac{e}{N}$. Instead of using the convergents of $\frac{e}{N}$, we will use the convergents of $\frac{e}{\phi_{0}}$ where $\phi_{0}$ is given by $\phi_{0}=N+1+\left(1+\frac{3 \sqrt{2}}{4}\right) N^{\frac{1}{2}}$. To this end, we will need the following result.

Lemma 1. For any $N>10^{6}$, we have

$$
\frac{\left(\frac{3}{\sqrt{2}}-2\right) N^{\frac{1}{2}}+2}{\left(N+2 N^{\frac{1}{2}}\right)^{2}}<\frac{1}{8 N^{\frac{3}{2}}} .
$$


Proof. Suppose that

$$
\frac{\left(\frac{3}{\sqrt{2}}-2\right) N^{\frac{1}{2}}+2}{\left(N+2 N^{\frac{1}{2}}\right)^{2}}<\frac{1}{8 N^{\frac{3}{2}}} .
$$

Then, clearing the denominators, we get

$$
8 N^{\frac{1}{2}}\left(\left(\frac{3}{\sqrt{2}}-2\right) N^{\frac{1}{2}}+2\right)<\left(N^{\frac{1}{2}}+2\right)^{2},
$$

which is equivalent to

$$
(12 \sqrt{2}-16) N+16 N^{\frac{1}{2}}<N+4 N^{\frac{1}{2}}+4 .
$$

This is true if

$$
(12 \sqrt{2}-16) N+16 N^{\frac{1}{2}}<N+4 N^{\frac{1}{2}},
$$

or equivalently $12<(17-12 \sqrt{2}) N^{\frac{1}{2}}$. This is valid if

$$
N>10^{6}>\left(\frac{12}{17-12 \sqrt{2}}\right)^{2} .
$$

This terminates the proof.

The following lemma is useful for approximating the sizes of the prime factors of an RSA modulus $N=p q$ when $p$ and $q$ are of the same bit-size.

Lemma 2. Let $N=p q$ be an RSA modulus with $q<p<2 q$. Then

$$
2 N^{\frac{1}{2}}<p+q<\frac{3 \sqrt{2}}{2} N^{\frac{1}{2}} .
$$

Proof. Assume that $q<p<2 q$. Then $1<\sqrt{\frac{p}{q}}<\sqrt{2}$, so, since the function $f(x)=x+\frac{1}{x}$ is increasing on $[1,+\infty)$, we get

$$
2<\sqrt{\frac{p}{q}}+\sqrt{\frac{q}{p}}<\sqrt{2}+\frac{1}{\sqrt{2}}=\frac{3 \sqrt{2}}{2} .
$$

If we multiply by $N^{\frac{1}{2}}$, we get

$$
2 N^{\frac{1}{2}}<p+q<\frac{3 \sqrt{2}}{2} N^{\frac{1}{2}}
$$

This terminates the proof.

Now, we present our first improved attack on KMOV based on the continued fraction algorithm. The following result shows that the secret information $p, q, d$ in a KMOV cryptosystem can be recovered from public information $(e, N)$. 
Theorem 6. Let $(N, e)$ be a public key in a KMOV cryptosystem with $N=$ $p q>10^{6}, q<p<2 q$ and $\operatorname{gcd}(e,(p+1)(q+1))$. If ed $\equiv 1(\bmod (p+1)(q+1))$ and $d<2 \sqrt{2} \frac{N^{\frac{3}{4}}}{\sqrt{e}}$, then one can factor $N$ in polynomial time in $\log (N)$.

Proof. Suppose that $N=p q$ with $q<p<2 q$. Then, by Lemma 2, we get

$$
N+1+2 N^{\frac{1}{2}}<(p+1)(q+1)<N+1+\frac{3 \sqrt{2}}{2} N^{\frac{1}{2}} .
$$

We set $\phi_{1}=N+1+2 N^{\frac{1}{2}}$ and $\phi_{2}=N+1+\frac{3 \sqrt{2}}{2} N^{\frac{1}{2}}$. Then $\left.(p+1)(q+1) \in\right] \phi_{1}, \phi_{2}[$. Let

$$
\phi_{0}=N+1+\left(1+\frac{3 \sqrt{2}}{4}\right) N^{\frac{1}{2}},
$$

be the midpoint of the interval $\left[\phi_{1}, \phi_{2}\right]$. Since $(p+1)(q+1) \in\left(\phi_{1}, \phi_{2}\right)$, then

$$
\left|(p+1)(q+1)-\phi_{0}\right| \leq \frac{1}{2}\left(\phi_{2}-\phi_{1}\right)
$$

If $e d \equiv 1(\bmod (p+1)(q+1))$, then $e d-k(p+1)(q+1)=1$, and

$$
\begin{aligned}
\left|\frac{e}{\phi_{0}}-\frac{k}{d}\right| & =\left|\left(\frac{e}{\phi_{0}}-\frac{e}{(p+1)(q+1)}\right)+\left(\frac{e}{(p+1)(q+1)}-\frac{k}{d}\right)\right| \\
& =\left|\frac{e\left((p+1)(q+1)-\phi_{0}\right)}{\phi_{0}(p+1)(q+1)}+\frac{1}{d(p+1)(q+1)}\right| \\
& =\left|\frac{e\left((p+1)(q+1)-\phi_{0}\right)}{\phi_{0}(p+1)(q+1)}+\frac{e}{(p+1)(q+1)(k(p+1)(q+1)+1)}\right| .
\end{aligned}
$$

Since $\phi_{0}(p+1)(q+1)>\phi_{1}^{2}$ and $(p+1)(q+1)(k(p+1)(q+1)+1)>\phi_{1}^{2}$, then

$$
\begin{aligned}
\left|\frac{e}{\phi_{0}}-\frac{k}{d}\right| & <e \frac{\frac{1}{2}\left(\phi_{2}-\phi_{1}\right)}{\phi_{1}^{2}}+e \frac{1}{\phi_{1}^{2}} \\
& =e \frac{\phi_{2}-\phi_{1}+2}{2 \phi_{1}^{2}} .
\end{aligned}
$$

Then, combining (3) and $\phi_{1}=N+1+2 \sqrt{N} \geq N+2 \sqrt{N}$, we get

$$
\left|\frac{e}{\phi_{0}}-\frac{k}{d}\right|<e \frac{\left(\frac{3 \sqrt{2}}{2}-2\right) \sqrt{N}+2}{2(N+2 \sqrt{N})^{2}} .
$$

Using Lemma 1, for $N>10^{6}$, we get

$$
\left|\frac{e}{\phi_{0}}-\frac{k}{d}\right|<\frac{e}{16 N^{\frac{3}{2}}} .
$$


Now, suppose that $\frac{e}{16 N^{\frac{3}{2}}}<\frac{1}{2 d^{2}}$, that is $d<\frac{2 \sqrt{2} N^{\frac{3}{4}}}{\sqrt{e}}$, then

$$
\left|\frac{e}{\phi_{0}}-\frac{k}{d}\right|<\frac{1}{2 d^{2}} .
$$

It follows by Theorem 4 that $\frac{k}{d}$ is a convergent of $\frac{e}{\phi_{0}}$ from which we deduce $k$ and $d$. Using the equation $e d-k(p+1)(q+1)=1$, we get $p+q=\frac{e d-1}{k}-N-1$ and combining with $N=p q$, we easily find $p$ and $q$. This gives to the factorization of $N=p q$. Notice that, since the continued fraction algorithm works in polynomial time, then finding $p$ and $q$ is done in polynomial time.

\subsection{Comparison with former attacks}

In [20], Pinch extended Wiener's attack [25] on RSA to KMOV and showed that one can factor the modulus $N=p q$ if the private exponent $d$ satisfies $d<\frac{1}{3} N^{\frac{1}{4}}$. In [1], Ibrahimpasic slightly extended the attack of Pinch by an extra exhaustive research. In both attacks, the bounds do not depend on the size of $e$. In our new attack, the bound is $d<2 \sqrt{2} \frac{N^{\frac{3}{4}}}{\sqrt{e}}$ and depends on $e$. In the typical situation where $e \approx N$, our bound becomes $d<2 \sqrt{2} N^{\frac{1}{4}} \approx 2.828 N^{\frac{1}{4}}$ while the bound in [20] is $d<\frac{1}{3} N^{\frac{1}{4}} \approx 0.333 N^{\frac{1}{4}}$. Observe that our new bound $d<2 \sqrt{2} \frac{N^{\frac{3}{4}}}{\sqrt{e}}$ is more significative for moderately small $e$.

Let us consider a numerical example. Consider the 1024 bit modulus $N$

$$
N=128072253291560984675731339942623874155571330351805615681477940
$$$$
737860111553200263411409851831323456088583497355190072283898949
$$

746366445389418926799490964902211240447125449181697155706714427

483626444781096408044876129844375261551528718257946239064984462 426873862229453485949998050716038824410982005466246527621,

and the 999 bit public exponent.

$e=296526935093015710407136686034981189608183689687233930438373260$

994003008667647176609955506859286957594312864516062333691708865

839614673732252521930067346220763331390433471403382719324360755

735108333314843772805919919463508848644534123617058298952149225

5372881221811248133999406005069737107180854644647.

Then, applying the continued fraction algorithm to $\frac{e}{\phi_{0}}$ and computing the convergents, the 130 th convergent is $\frac{k}{d}$ where

$$
\begin{aligned}
k= & 439246113481593542214907254431461323475931905724710548163257922 \\
& 73119943084, \\
d= & 189713759006418854581978701838234268226797542876185500122247305 \\
& 6385648715923809927 .
\end{aligned}
$$


Using this convergent, we get $p+q=\frac{e d-1}{k}$. Then combining with $p q=N$, we get

$$
\begin{aligned}
p= & 122295652435077729919345520517086986879675097236430221980450907 \\
& 006278884550539602496027848592931847870084590996181730049111792 \\
& 44406300082071971851405178417, \\
q= & 104723471964426405086080002568566304601367956338101575543737215 \\
& 792893331651240463496547152238295322902114471979717345643807495 \\
& 25832667841702102917782974613 .
\end{aligned}
$$

We notice that $\frac{k}{d}$ is not among the convergents of $\frac{e}{N}$ which implies that the methods of Pinch and Ibrahimpasic will not succeed.

\section{A New Improved Attack Based on Coppersmith's Method}

In this section, we present a new attack on KMOV based on Coppersmith's method.

\subsection{The new attack}

Theorem 7. Let $(N, e)$ be a public key for the KMOV cryptosystem where $N=$ $p q$ is an RSA modulus and $e=N^{\beta}$. Suppose that e satisfies the equation eu$(p+1)(q+1) v=w$ with $u<N^{\delta}$ and $|w|<N^{\gamma}$. If

$$
\delta<\frac{7}{6}-\gamma-\frac{1}{3} \sqrt{6 \beta-6 \gamma+1}-\varepsilon,
$$

for a small positive constant $\varepsilon$, then one can factor $N$ in polynomial time.

Proof. Suppose that $N=p q$ is an RSA modulus and $e$ is a public exponent satisfying $e u-(p+1)(q+1) v=w$. Since $(p+1)(q+1)=N+p+q+1$, then $v(N+p+q+1)+w \equiv 0(\bmod e)$, which can be rewritten as $v(p+q+1)+N v+w \equiv$ $0(\bmod e)$. Consider the polynomial $f(x, y, z)=x y+N x+z$, Then $(x, y, z)=$ $(v, p+q+1, w)$ is a solution of the modular polynomial equation $f(x, y, z) \equiv 0$ $(\bmod e)$. To find the solution $(v, p+q+1, w)$, we apply Coppersmith's method $[8]$. Let $m$ and $t$ be two positive integers to be optimized later. We use $f(x, y, z)$ to build the sets of polynomials

$$
\begin{aligned}
& G_{k, i_{1}, i_{2}, i_{3}}(x, y, z)=x^{i_{1}-k} z^{i_{3}} f(x, y, z)^{k} e^{m-k} \\
& \text { for } \quad k=0, \ldots m, i_{1}=k, \ldots, m, i_{2}=k, i_{3}=m-i_{1}, \\
& H_{k, i_{1}, i_{2}, i_{3}}(x, y, z)=y^{i_{2}-k} z^{i_{3}} f(x, y, z)^{k} e^{m-k} \\
& \quad \text { for } \quad k=0, \ldots m, i_{1}=k, i_{2}=k+1, \ldots, i_{1}+t, i_{3}=m-i_{1} .
\end{aligned}
$$

Let $\mathcal{L}$ denote the lattice spanned by the coefficient vectors of the polynomials $G_{k, i_{1}, i_{2}, i_{3}}(X x, Y y, Z z)$ and $H_{k, i_{1}, i_{2}, i_{3}}(X x, Y y, Z z)$. By choosing the increasing 
ordering following the $i_{1}$ 's, then the $i_{2}$ 's, and the $i_{3}$ 's, one find a left triangular matrix. For $m=2$ and $t=1$, the coefficient matrix for $\mathcal{L}$ is presented in Table 1 where the monomials are

$$
\left\{z^{3}, x z^{2}, x^{2} z, x^{3}, x y z^{2}, x^{2} y z, x^{3} y, x^{2} y^{2} z, x^{3} y^{2}, x^{3} y^{3}, x y^{2} z^{2}, x^{2} y^{3} z, x^{2} y z, x^{3} y^{4}\right\} .
$$

The non-zero elements are marked with an ' $\circledast$ ' and do not influence the value of the determinant.

\begin{tabular}{|c|c|c|c|c|c|c|c|c|c|c|}
\hline & $z^{3}$ & $x z^{2}$ & $x^{2} z$ & $x^{3}$ & $x y z^{2}$ & $x^{2} y z$ & $x^{3} y$ & $x^{2} y^{2} z$ & $x^{3} y^{2}$ & $x^{3} y^{3}$ \\
\hline$G_{k, i_{1}, i_{2}, i_{3}}$ & & & & & & & & & & \\
\hline$G_{0,0,0,3}$ & $z^{3} e^{3}$ & 0 & 0 & 0 & 0 & 0 & 0 & 0 & 0 & 0 \\
\hline$G_{0,1,0,2}$ & 0 & $X z^{2} e^{3}$ & 0 & 0 & 0 & 0 & 0 & 0 & 0 & 0 \\
\hline$G_{0,2,0,1}$ & 0 & 0 & $X^{2} Z e^{3}$ & 0 & 0 & 0 & 0 & 0 & 0 & 0 \\
\hline$G_{0,3,0,0}$ & 0 & 0 & 0 & $x^{3}$ & 0 & 0 & 0 & 0 & 0 & 0 \\
\hline$G_{1,1,1,2}$ & $\circledast$ & 0 & 0 & 0 & $X Y Z^{2} e^{2}$ & 0 & 0 & 0 & 0 & 0 \\
\hline$G_{1,2,1,1}$ & 0 & $\circledast$ & $\circledast$ & 0 & 0 & $X^{2} Y Z e^{2}$ & 0 & 0 & 0 & 0 \\
\hline$G_{1,3,1,0}$ & 0 & 0 & $\circledast$ & $\circledast$ & 0 & 0 & $X^{3} Y e^{2}$ & 0 & 0 & 0 \\
\hline$G_{2,2,2,1}$ & $\circledast$ & $\circledast$ & $\circledast$ & 0 & $\circledast$ & $\circledast$ & 0 & $X^{2} Y^{2} Z e$ & 0 & 0 \\
\hline$G_{2,3,2,0}$ & 0 & $\circledast$ & $\circledast$ & $\circledast$ & 0 & $\circledast$ & $\circledast$ & 0 & $X^{3} Y^{2} e$ & 0 \\
\hline$G_{3,3,3,0}$ & $\circledast$ & 0 & $\circledast$ & $\circledast$ & $\circledast$ & $\circledast$ & $\circledast$ & $\circledast$ & $\circledast$ & $X^{3} Y^{3}$ \\
\hline $\begin{array}{c}H_{k, i_{1}, i_{2}, i_{3}} \\
H_{0,0,1,3}\end{array}$ & 0 & 0 & 0 & 0 & $\circledast$ & 0 & 0 & 0 & 0 & $\circledast$ \\
\hline $\begin{array}{l}H_{1,1,2,2}, 1,3 \\
H_{1,1}\end{array}$ & 0 & 0 & 0 & 0 & $\circledast$ & $\circledast$ & 0 & $\circledast$ & 0 & 0 \\
\hline$H_{2,2,3,1}$ & 0 & 0 & 0 & $\circledast$ & 0 & 0 & $\circledast$ & $\circledast$ & 0 & 0 \\
\hline$H_{3,3,4,0}$ & 0 & 0 & 0 & 0 & $\circledast$ & 0 & 0 & $\circledast$ & $\circledast$ & 0 \\
\hline
\end{tabular}

\begin{tabular}{||c||c|c|c|c||}
\hline & $x y^{2} z^{2}$ & $x^{2} y^{3} z$ & $x^{2} y z$ & $x^{3} y^{4}$ \\
\hline \hline$G_{k, i_{1}, i_{2}, i_{3}}$ & 0 & 0 & 0 & 0 \\
$G_{0,0,0,3}$ & 0 & 0 & 0 & 0 \\
$G_{0,1,0,2}$ & 0 & 0 & 0 & 0 \\
$G_{0,2,0,1}$ & 0 & 0 & 0 & 0 \\
$G_{0,3,0,0}$ & 0 & 0 & 0 & 0 \\
$G_{1,1,1,2}$ & 0 & 0 & 0 & 0 \\
$G_{1,2,1,1}$ & 0 & 0 & 0 & 0 \\
$G_{1,3,1,0}$ & 0 & 0 & 0 & 0 \\
$G_{2,2,2,1}$ & 0 & 0 & 0 & 0 \\
$G_{2,3,2,0}$ & 0 & 0 & 0 & 0 \\
$G_{3,3,3,0}$ & 0 & 0 & 0 & 0 \\
$H_{k, i_{1}, i_{2}, i_{3}}$ & & & 0 \\
$H_{0,0,1,3}$ & $X Y^{2} Z^{2} e^{2}$ & 0 & 0 & 0 \\
$H_{1,1,2,2}$ & $\circledast$ & $X^{2} Y^{3} Z e$ & 0 & 0 \\
$H_{2,2,3,1}$ & 0 & 0 & $X^{2} Y Z e$ & 0 \\
$H_{3,3,4,0}$ & 0 & 0 & 0 & $X^{3} Y^{4}$ \\
\hline
\end{tabular}

Table 1. The coefficient matrix for the case $m=2, t=1$.

The determinant of the triangular matrix is then the determinant of the lattice $\mathcal{L}$ and can be easily computed as

$$
\operatorname{det}(\mathcal{L})=e^{n_{e}} X^{n_{X}} Y^{n_{Y}} Z^{n_{Z}} .
$$

To find the values of the exponents $n_{e}, n_{X}, n_{Y}, n_{Z}$, define the sum $S(a)$ by

$$
S(a)=\sum_{k=0}^{m} \sum_{i_{1}=k}^{m} \sum_{i_{2}=k}^{k} \sum_{i_{3}=m-i_{1}}^{m-i_{1}} a+\sum_{k=0}^{m} \sum_{i_{1}=k}^{k} \sum_{i_{2}=k+1}^{i_{1}+t} \sum_{i_{3}=m-i_{1}}^{m-i_{1}} a .
$$


By the construction of the polynomials $G$ and $H$, we get

$$
\begin{aligned}
& n_{e}=S(m-k)=\frac{1}{6} m(m+1)(2 m+3 t+4), \\
& n_{X}=S\left(i_{1}\right)=\frac{1}{6} m(m+1)(2 m+3 t+4), \\
& n_{Y}=S\left(i_{2}\right)=\frac{1}{6}(m+1)\left(m^{2}+3 m t+3 t^{2}+2 m+3 t\right), \\
& n_{Z}=S\left(i_{3}\right)=\frac{1}{6} m(m+1)(m+3 t+2) .
\end{aligned}
$$

The dimension of the lattice is the number of rows in the matrix. It can be estimated as

$$
\omega=S(1)=\frac{1}{2}(m+1)(m+2 t+2) .
$$

If we set $t=\tau m$ for some positive $\tau$, then the dominant terms of the exponents in (5) and 6 are

$$
\begin{aligned}
n_{e} & \approx \frac{1}{6}(3 \tau+2) m^{3}+o\left(m^{3}\right), \\
n_{X} & \approx \frac{1}{6}(3 \tau+2) m^{3}+o\left(m^{3}\right), \\
n_{Y} & \approx \frac{1}{6}\left(3 \tau^{2}+3 \tau+1\right) m^{3}+o\left(m^{3}\right), \\
n_{Z} & \approx \frac{1}{6}(3 \tau+1) m^{3}+o\left(m^{3}\right), \\
w & \approx \frac{1}{6}(6 \tau+3) m^{2}+o\left(m^{2}\right) .
\end{aligned}
$$

Next, we apply the LLL algorithm 2 to the lattice $\mathcal{L}$. We then get a reduced basis where the three first vectors $h_{i}, i=1,2,3$ satisfy

$$
\left\|h_{1}\right\| \leq\left\|h_{2}\right\| \leq\left\|h_{3}\right\| \leq 2^{\frac{\omega(\omega-1)}{4(\omega-2)}} \operatorname{det}(\mathcal{L})^{\frac{1}{\omega-2}} .
$$

To apply Howgrave-Graham's Theorem 1 to $h_{1}, h_{2}$ and $h_{3}$, we set

$$
2^{\frac{\omega(\omega-1)}{4(\omega-2)}} \operatorname{det}(\mathcal{L})^{\frac{1}{\omega-2}}<\frac{e^{m}}{\sqrt{\omega}},
$$

from which we deduce

$$
\operatorname{det}(\mathcal{L})<2^{-\frac{\omega(\omega-1)}{4}} \frac{1}{(\sqrt{\omega})^{\omega-2}} e^{m(\omega-2)} .
$$

Using (4), we get

$$
e^{n_{e}} X^{n_{X}} Y^{n_{Y}} Z^{n_{Z}}<2^{-\frac{\omega(\omega-1)}{4}} \frac{1}{(\sqrt{\omega})^{\omega-2}} e^{m(\omega-2)} .
$$


Suppose that $e=N^{\beta}, u<N^{\delta}$ and $|w|<N^{\gamma}$. Then, using Lemma 2, we have $p+q+1 \leq 2 p<2 \sqrt{2} \sqrt{N}$. Since $p+q+1$ is represented by $y$, we set $Y=\lfloor 2 \sqrt{2} \sqrt{N}\rfloor$. On the other hand, since $(p+1)(q+1)>N$ and $|w|<e u$, we get

$$
|v|=\frac{|e u-w|}{(p+1)(q+1)}<\frac{e u+|w|}{(p+1)(q+1)}<\frac{2 e u}{N}<2 N^{\beta+\delta-1} .
$$

Since $v$ is represented by $x$, we set $X=\left\lfloor 2 N^{\beta+\delta-1}\right\rfloor$. Also, since $w$ is represented by $Z$, we set $Z=\left\lfloor N^{\gamma}\right\rfloor$. It follows that the solution $(x, y, z)=(v, p+q+1, w)$ satisfies $|x|<X,|y|<Y$ and $|z|<Z$ and $(8)$ is satisfied if

$$
2^{n_{X}}(2 \sqrt{2})^{n_{Y}} N^{n_{e} \beta+n_{X}(\beta+\delta-1)+\frac{n_{Y}}{2}+n_{Z} \gamma}<2^{-\frac{\omega(\omega-1)}{4}} \frac{1}{(\sqrt{\omega})^{\omega-2}} N^{m(\omega-2) \beta} .
$$

Using the approximations of $n_{e}, n_{X}, n_{Y}, n_{Z}$ given in (7) and $\omega$ given 6, the inequality 8 leads to

$$
\begin{aligned}
& N^{\left((3 \tau+2) \beta+(3 \tau+2)(\beta+\delta-1)+\frac{3 \tau^{2}+3 \tau+1}{2}+(3 \tau+1) \gamma\right) m^{3}} \\
& \quad<2^{-n_{X}}(2 \sqrt{2})^{-n_{Y}} 2^{-\frac{\omega(\omega-1)}{4}} \frac{1}{(\sqrt{\omega})^{\omega-2}} N^{-2 \beta m} N^{(6 \tau+3) \beta m^{3}} .
\end{aligned}
$$

To homogenize the exponentiation of $N$, we set

$$
2^{-n_{X}}(2 \sqrt{2})^{-n_{Y}} 2^{-\frac{\omega(\omega-1)}{4}} \frac{1}{(\sqrt{\omega})^{\omega-2}} N^{-2 \beta m}=N^{-\mu m^{3}}
$$

where $\mu$ is a small positive constant. Then, taking logarithms and dividing by $m^{3} \log N$, we get

$$
(3 \tau+2) \beta+(3 \tau+2)(\beta+\delta-1)+\frac{3 \tau^{2}+3 \tau+1}{2}+(3 \tau+1) \gamma-(6 \tau+3) \beta<-\mu
$$

The optimal value for the left hand side is $\tau_{0}=\frac{1-2 \delta-2 \gamma}{2}$, which, plugged in the former inequality leads to

$$
-12 \delta^{2}-24 \delta \gamma-12 \gamma^{2}+8 \beta+28 \delta+20 \gamma-15<-8 \mu
$$

and consequently

$$
\delta<\frac{7}{6}-\gamma-\frac{1}{3} \sqrt{6 \beta-6 \gamma+1}-\varepsilon
$$

where $\varepsilon$ is a small positive constant that depends on $m$ and $N$. Within this condition, the reduced lattice has three polynomials $h_{1}(x, y, z), h_{2}(x, y, z)$ and $h_{2}(x, y, z)$ sharing the root $\left(x_{0}, y_{0}, z_{0}\right)=(v, p+q+1, w)$. Then, applying Gröbner basis or resultant computations, we get the expected solution $\left(x_{0}, y_{0}, z_{0}\right)$ from which we deduce $p+q=y-1$. Together with the equation $p q=N$, this leads to finding $p$ and $q$. This terminates the proof. 


\subsection{Comparison with former attacks}

In [18, Nitaj presented an algorithm for factoring the modulus $N=p q$ when the public exponent $e$ satisfies an equation of the form $e u-(p+1)(q+1) v=w$, where the unknown parameters $u, v$ and $w$ are such that

$$
|w|<\frac{(p-q) N^{\frac{1}{4}} v}{3(p+q)}, \quad u v<\frac{\sqrt{2} \sqrt{N}}{12} .
$$

The idea in 18] is to compute the convergents of the continued fraction of $\frac{e}{N}$, and for each convergent $\frac{v}{u}$ with $u v<\frac{\sqrt{2} \sqrt{N}}{12}$, to compute $U$ and $V$ with

$$
U=\frac{e u}{v}-N-1, \quad V=\sqrt{\left|U^{2}-4 N\right|} .
$$

Then $\tilde{p}=\frac{1}{2}(U+V)$ is a possible approximation of the prime factor $p$ with error term of at most $2 N^{\frac{1}{4}}$. If so, then by applying Coppersmith's method, one can find $p$, and then factor $N$.

To compare our new results and the result of [18, suppose that $e=N^{\beta}$, $u<N^{\delta}$ and $|w|<N^{\gamma}$. Then, by (9), we get $|v|<2 N^{\beta+\delta-1}$. Hence, the inequalities (12) are fulfilled if

$$
N^{\gamma}<\frac{2(p-q) N^{\frac{1}{4}} N^{\beta+\delta-1}}{3(p+q)}, \quad 2 N^{\delta} N^{\beta+\delta-1}<\frac{\sqrt{2} \sqrt{N}}{12} .
$$

Then, neglecting the constants and assuming that $p-q \approx p+q$, the former two inequalities are true if

$$
\gamma<\frac{1}{4}+\beta+\delta-1, \quad 2 \delta+\beta-1<\frac{1}{2} .
$$

This leads to $\delta<\frac{3}{4}-\frac{1}{2} \beta$, which is to be compared with the new bound

$$
\delta<\frac{7}{6}-\gamma-\frac{1}{3} \sqrt{6 \beta-6 \gamma+1}-\varepsilon .
$$

Define

$$
\delta_{0}=\frac{3}{4}-\frac{1}{2} \beta, \quad \delta_{1}=\frac{7}{6}-\gamma-\frac{1}{3} \sqrt{6 \beta-6 \gamma+1} .
$$

A typical situation is when $e \approx N$, that is $\beta=1$, and $|w|$ is small, that is $\gamma=0$. Then the bounds $\delta_{0}$ and $\delta_{1}$ are $\delta_{0}=0.25, \delta_{1} \approx 0.284$. We see that the new method overcome the method of $[18$ in the most realistic situations of instances of KMOV.

\section{Conclusion}

We have presented two new attacks on the KMOV cryptosystem which is an RSA type cryptosystem based on elliptic curves. The first attack is based on the continued fraction algorithm and the second is based on Coppersmith's method. Both attacks work when the private key is suitably small and the new results improve the former attacks on the KMOV elliptic curve cryptosystem. 


\section{References}

1. D. Boneh, Twenty years of attacks on the RSA cryptosystem, Notices of the American Mathematical Society 46(2), 203-213, 1999.

2. D. Boneh, G. Durfee, Cryptanalysis of RSA with private key d less than $N^{0.292}$, IEEE Transactions on Information Theory 46, 1339-1349, 2000.

3. M. Bunder, J. Tonien, A new improved attack on RSA, in Proceedings of the 5th International Cryptology and Information Security Conference, 2016, pp. 101-110.

4. M. Bunder, A. Nitaj, W. Susilo, J. Tonien, A new attack on three variants of the RSA cryptosystem, in: Proceedings of ACISP 2016, Lecture Notes in Computer Science 9723, 2016, pp. 258-268.

5. M. Bunder, A. Nitaj, W. Susilo, J. Tonien, A generalized attack on RSA type cryptosystems, Theoretical Computer Science 704, 74-81, 2017.

6. M. Bunder, J. Tonien, A new attack on the RSA cryptosystem based on continued fractions, Malaysian Journal of Mathematical Sciences 11(S3), 45-57, 2017.

7. M. Bunder, A. Nitaj, W. Susilo, J. Tonien, Cryptanalysis of RSA-type cryptosystems based on Lucas sequences, Gaussian integers and elliptic curves, Journal of Information Security and Applications 40, 193-198, 2018.

8. D. Coppersmith, Small solutions to polynomial equations, and low exponent RSA vulnerabilities, Journal of Cryptology 10(4), 233-260, 1997.

9. G.H. Hardy and E.M. Wright, An Introduction to the Theory of Numbers, Oxford University Press, London, 1965.

10. N. Howgrave-Graham, Finding small roots of univariate modular equations revisited, in: Proceedings of IMA International Conference on Cryptography and Coding 1997, Lecture Notes in Computer Science 1355, 1997, pp. 131-142.

11. B. Ibrahimpasic, Cryptanalysis of KMOV cryptosystem with short secret exponent, in: Proceedings of Central European Conference on Information and Intelligent Systems, 2008.

12. E. Jochemsz, A. May, A strategy for finding roots of multivariate polynomials with new applications in attacking RSA variants, in: Proceedings of ASIACRYPT 2006, Lecture Notes in Computer Science 4284, 2006, pp. 267-282.

13. N. Koblitz, Elliptic curve cryptosystems, Mathematics of Computation 48, 203209, 1987.

14. K. Koyama, U. M. Maurer, T. Okamoto and S. A. Vanstone, New public-key schemes based on elliptic curves over the ring $\mathbb{Z}_{n}$, in: Proceedings of CRYPTO 1991, Lecture Notes in Computer Science 576, 1991, pp. 252-266.

15. A.K. Lenstra, H.W. Lenstra, L. Lovász, Factoring polynomials with rational coefficients, Mathematische Annalen 261, 513-534, 1982.

16. A. May, New RSA Vulnerabilities using Lattics Reduction Methods, Ph.D. Dissertation. University of Paderborn, (2003) http://www.cits.rub.de/imperia/md/ content/may/paper/bp.ps

17. V.S. Miller, Use of elliptic curves in cryptography, in: Proceedings of CRYPTO 1985, Lecture Notes in Computer Science 218, 1986, pp. 417-426.

18. A. Nitaj, A new attack on the KMOV cryptosystem, Bulletin of the Korean Mathematical Society 51 (5), 1347-1356, 2014.

19. A. Nitaj, Y. Pan, J. Tonien, A Generalized Attack on Some Variants of the RSA Cryptosystem, in: Proceedings of SAC 2018, Lecture Notes in Computer Science 11349, 2019, pp. 421-433.

20. R.G.E. Pinch, Extending the Wiener attack to RSA-type cryptosystems, Electronics Letters 31 (20), 1736-1738, 1995. 
21. R. Rivest, A. Shamir, L. Adleman, A Method for Obtaining digital signatures and public-key cryptosystems, Communications of the ACM 21 (2), 120-126, 1978.

22. S. Schmitt, H.G. Zimmer, Elliptic curves. A computational approach. Walter de Gruyter, Berlin, 2003.

23. J.H. Silverman, The Arithmetic of Elliptic Curves, Graduate Texts in Mathematics 106, Springer-Verlag, 1986.

24. W. Susilo, J. Tonien, G, Yang, The Wiener Attack on RSA Revisited: A Quest for the Exact Bound, in: Proceedings of ACISP 2019, Lecture Notes in Computer Science 11547, 2019, pp. 381-398.

25. M. Wiener, Cryptanalysis of short RSA secret exponents, IEEE Transactions on Information Theory 36, 553-558, 1990. 